\title{
The safety of using anti-VEGF: Is there strength in numbers? Curtis LH, Hammill BG, Schulman KA, Cousins SW (2010) Risks of mortality, myocardial infarction, bleeding, and stroke associated with therapies for age-related macular degeneration. Arch Ophthalmol 128(10):1273-1279
}

\author{
David Wong • Antonia M. Joussen
}

Received: 10 December 2010 / Accepted: 10 December 2010/Published online: 6 January 2011

(C) The Author(s) 2010. This article is published with open access at Springerlink.com

\section{Keywords Anti-VEGF}

The safety of using anti-VEGF remains a hot topic. There are not many retinal specialists who have not "fought" at one stage or another and tried to implement anti-VEGF into their practice. Cost is a big issue. Nationally, some countries have literally taken years to deliberate as to whether ranibizumab is cost-effective. For example, in the United Kingdom, the National Institute of Clinical Excellence only came to its "final appraisal determination" in 2008 [1], when the drug had been commercially available for some years. Other countries have yet to decide. In China, the Xinhau News Agency reported an incident where 61 out of 116 patients developed serious adverse reactions after receiving bevacizumab injection in Shanghai No 1 People's Hospital [2]. This reverberated around Asia, and raised again the issue of who is liable when drugs are used off-label. The uncertainty while we await the results of head-to-head

\section{A. M. Joussen}

Department of Ophthalmology Charité,

University Medicine Berlin,

Virchowklinikum (CVK), Augustenburger Platz 1,

13353 Berlin, Germany

\section{A. M. Joussen}

Klinikum Steglitz (CBF), Hindenburgdamm 30,

12200 Berlin, Germany

\section{Wong $(\bowtie)$}

The Eye Institute, LKS Faculty of Medicine,

The University of Hong Kong,

Hong Kong, People's Republic of China

e-mail: shdwong@hku.hk randomised trials between ranibizumab and bevacizumab has led some national health services to choose pegaptanib as the safest, affordable and approved first-line drug for the treatment of macular degeneration.

In the October issue of the Archives of Ophthalmology, Curtis et al. published an article on the risks of mortality, myocardial infarction, bleeding, and stroke associated with therapies for age-related macular degeneration (AMD) [3]. This is a retrospective study involving 146,942 patients identified from the Centers for Medicare \& Medicaid Services for all Medicare beneficiaries from January 1 2004 through December 31 2007. Medicare-based studies have a long track record of finding significant associations. For example, in 1992 Javitt et al. studied 57,103 cases of extracapsular cataract extraction, and identified that patients who subsequently underwent capsulotomy had a 4-fold increase in risk of developing retinal detachment. This was the first time an incontrovertible link had been established between yag laser capsulotomy and retinal detachment. There is, therefore, a real strength in numbers. The weight of evidence can definitively resolve some controversial issues. Does the current study resolve once and for all the safety issue with regard to anti-VEGF?

In this study, patients were divided into four groups, depending on their initial treatment, namely photodynamic therapy (PDT), pegaptanib, bevacizumab and ranibizumab. Four adverse events were examined: all-cause mortality, incident myocardial infarction, bleeding, and incident stroke. Data from patients who received a different therapy from the one they received initially were censored. Naturally, the number of patients that received PDT and pegaptanib declined throughout the period of the study. 
Surprisingly, there were small differences in the 1-year cumulative incidence of adverse event by treatment group. There were statistically significant differences in mortality; among beneficiaries who received pegaptanib, $4.8 \%$ died compared with $4.4 \%$ in the bevacizumab group and $4.1 \%$ in the PDT and ranibizumab groups. The cumulative incidence of myocardial infarction was slightly higher in the PDT and pegaptanib groups than in the bevacizumab and ranibizumab groups, but the overall $P$ value did not reach statistical significance. There was no statistically significant relationship between treatment group and bleeding events or stroke. Even after adjusting for confounding factors such as age, gender, race/ethnicity and co-morbidity, significant differences existed. The hazard of mortality was significantly lower with ranibizumab use than with PDT or pegaptanib use. The hazard of myocardial infarction was significantly lower with ranibizumab use than with PDT. There were no significant differences in the hazard of mortality or myocardial infarction between bevacizumab use and other treatments.

What is more interesting is the comparison between bevacizumab and ranibizumab. A subgroup analysis was made of 19,026 patients who received ranibizumab and 21,815 who received bevacizumab as first-line therapy. After adjustment for baseline characteristics and co-morbid conditions, the hazards of mortality and stroke (hazard ratio of 0.86 and 0.78 ) were significantly lower with ranibizumab therapy than with bevacizumab therapy. The authors thought that patients with higher socioeconomic status may have been more likely to receive ranibizumab vs bevacizumab, so the primary analysis may have been subject to selection bias. Therefore, in a secondary analysis, the study population was limited to patients who received ranibizumab or bevacizumab in medical practices that basically used either bevacizumab or ranibizumab exclusively during the third or fourth quarter of 2006. The results then show no difference.

There are always limitations with this type of study. The large number involved means that many significant associations can be discovered. However, one should appreciate the result merely points to association and not necessary causation. We do not know if other factors such as socioeconomic status caused bias. It is always easy to say that a randomised control trial will resolve the matter definitively. The authors pointed out, "The National Eye Institute-sponsored comparative trial of the efficacy of ranibizumab and bevacizumab will randomly assign 1,200 patients with newly diagnosed age-related macular degeneration to one of four treatment groups (two bevacizumab regimens and two ranibizumab regimens). Given the baseline risks of thromboembolic events in this population, the sample size needed to detect even a $50 \%$ relative increase in risk (from $2.0 \%$ to $3.0 \%$ ) would be at least an order of magnitude larger than the trial protocol dictates. As designed, the trial will not adequately assess the relative safety of the two therapies."

The take-home measure for me personally is that the risk overall with anti-VEGF use is small, and comparable with that for PDT. Within the limitations of the study, it is not unreasonable to think that the selective anti-VEGF is not superior in its safety profile compared to pan anti-VEGF agents. The small difference between bevacizumab and ranibizumab might well be spurious, but will no doubt fuel more discussions.

Open Access This article is distributed under the terms of the Creative Commons Attribution Noncommercial License which permits any noncommercial use, distribution, and reproduction in any medium, provided the original author(s) and source are credited.

\section{References}

1. NICE Technology Appraisal 155, Issued August 2008. Pegaptanib and ranibizumab for the treatment of age-related macular degeneration.

2. http://news.xinhuanet.com/english2010/china/2010-09/15/ c $13495920 . \mathrm{htm}$ accessed on 9 December 2010

3. Curtis LH, Hammill BG, Schulman KA, Cousins SW (2010) Risks of mortality, myocardial infarction, bleeding, and stroke associated with therapies for age-related macular degeneration. Arch Ophthalmol 128(10):1273-1279 